\title{
Abordagem multiprofissional da criança com dor musculoesquelética
}

\author{
Multiprofessional assistance of children with musculoskeletal pain
}

Melissa Mariti Fraga' ${ }^{1}$ Claudio Arnaldo Len²

A fibromialgia juvenil (FMJ) é uma doença de caráter crônico ou recorrente, pouco reconhecida na faixa etária pediátrica. No entanto, destaca-se entre as doenças reumáticas devido ao impacto negativo na qualidade de vida das crianças, dos adolescentes e das suas famílias. O seu tratamento é complexo e os medicamentos habitualmente utilizados para o controle da dor musculoesquelética, como os analgésicos e os anti-inflamatórios não hormonais, têm pouca eficácia, além dos efeitos adversos já conhecidos pelos pediatras $^{(1,2)}$.

A busca das melhores evidências para o tratamento da FMJ tem mostrado que são obtidos bons resultados nos casos em que os pacientes são avaliados e tratados por uma equipe multiprofissional, composta por médicos, fisioterapeutas e psicólogos, entre outros ${ }^{(3)}$, com envolvimento dos pacientes e seus pais. Dentre as modalidades terapêuticas, destacam-se a prática regular de exercícios aeróbicos ${ }^{(4)}$ (que deve ser fortemente estimulada) e a psicoterapia cognitivo-comportamental ${ }^{(5)}$. Outras técnicas classicamente utilizadas para o controle da dor podem ser aplicadas em alguns pacientes, com resultados promissores. Dentre elas, destacam-se a acupuntura, o biofeedback, além de técnicas especializadas em dessensibilização, ainda pouco difundidas em nosso meio ${ }^{(6-8)}$.

$\mathrm{Na}$ publicação pioneira de Dias $e t{ }^{(9)}$, são descritos os resultados obtidos por meio do seguimento regular de pacientes com FMJ tratados por acupuntura - método milenar e tradicional da medicina chinesa. Nesta série de casos, os autores observaram uma melhora significativa da dor musculoesquelética, mensurada por escalas de dor e pelo número de pontos dolorosos. Além do benefício direto da acupuntura na dor, não foram observados efeitos adversos, o que é um ponto muito importante por se tratarem de pacientes com

'Pós-graduanda do Programa de Pediatria e Ciências Aplicadas à Pediatria da Escola Paulista de Medicina da Universidade Federal de São Paulo (Unifesp); Médica assistente do Ambulatório de Dor Musculoesquelética do Setor de Reumatologia do Departamento de Pediatria da Escola Paulista de Medicina da Unifesp, São Paulo, SP, Brasil

2Doutor em Ciências; Professor Adjunto do Setor de Reumatologia do Departamento de Pediatria da Escola Paulista de Medicina da Unifesp, São Paulo, SP, Brasil idades entre dez e 18 anos, em plena fase de crescimento e desenvolvimento.

A pesquisa em acupuntura apresenta um desafio metodológico. Enfrentam-se obstáculos de caráter científico, como a formação de um grupo placebo adequado, uma vez que se estuda o efeito da inserção de agulhas. Escolher um método para simular o agulhamento sem a penetração da pele (para não interferir no objetivo do estudo) é fundamental. Outro obstáculo é a impossibilidade de realizar estudo duplo-cego, uma vez que o médico acupunturista saberá se o paciente recebeu ou não o agulhamento. A seleção do grupo a ser estudado é também motivo de discussão, pois, na acupuntura, opta-se pelo tratamento do paciente com seu consentimento juntamente com o de seu responsável. Cabe destacar que no estudo de Dias et $a l^{(9)}$, tais aspectos éticos foram respeitados.

A acupuntura é uma modalidade da Medicina Tradicional Chinesa que surgiu há mais de 5.000 anos. Foi considerada como especialidade médica pela Associação Médica Brasileira, em 1995, com a realização da primeira prova de título para a especialidade em 1999. Está inserida no Sistema Único de Saúde desde 1988. Baseia-se na hipótese de que a energia $(Q i)$ circula pelo corpo em canais ou meridianos. Se esta energia flui livremente, o paciente está saudável, porém se, por qualquer razão, tal fluxo é bloqueado, o paciente poderá ter dor ou adoecer. A inserção de agulhas em pontos específicos predeterminados do corpo restitui o livre fluxo da energia com o objetivo de melhorar a condição de saúde do indivíduo globalmente.

Atualmente, a principal indicação da acupuntura é o tratamento da dor, mas pode ser utilizada na terapia da asma, enurese noturna, paralisia cerebral, diminuição dos
Endereço para correspondência:

Prof. Dr. Claudio A. Len

Avenida Brigadeiro Faria Lima, 1.826 cj 205

CEP 01451-001 - São Paulo/SP

E-mail: claudiolen@gmail.com

Conflito de interesse: nada a declarar 
sintomas do tratamento oncológico, entre outras. Dentre as técnicas utilizadas, destacam-se a estimulação manual, elétrica (eletroacupuntura) ou pelo calor (moxabustão) das agulhas já inseridas. Nos pacientes pediátricos, pode-se também realizar o aquecimento da região do ponto de acupuntura com bastão de ervas (Artemisia vulgaris), sem o uso de agulhas. Outra técnica empregada na pediatria é a de colocar sementes nos pontos de acupuntura do pavilhão auricular sem agulhas.

Com relação ao mecanismo de ação da acupuntura, há liberação de neurotransmissores que atuam na segunda camada do corno dorsal e, com a liberação de encefalina, ocorre o bloqueio efetivo de qualquer transmissão de dor através das fibras C. Quanto ao procedimento, sabe-se que o medo da agulha por parte dos pacientes pediátricos e de suas famílias é uma realidade. As crianças são fortemente influenciadas pelo estado emocional dos pais, muitas vezes refletindo suas angústias, dúvidas e medos. Pode-se contornar esta situação fazendo os pais participarem de maneira ativa do tratamento. A explicação do procedimento para a criança e seus pais, a presença dos pais ao lado do paciente durante toda a sessão, as mãos aquecidas do médico acupunturista,

\section{Referências bibliográficas}

1. Lanas A, Sopeña F. Nonsteroidal anti-inflammatory drugs and lower gastrointestinal complications. Gastroenterol Clin North Am 2009;38:333-52.

2. Len C, Hilario MO, Kawakami E, Terreri MT, Becker DJ, Goldenberg J et al. Gastroduodenal lesions in children with juvenile rheumatoid arthritis. Hepatogastroenterology 1999;46:991-6.

3. Molina J, Silva SG, Teles FM, Fraga MM, Paulo LT, Bugni V et al. Diffuse idiopathic musculoskeletal pain in childhood and adolescence. Rev Paul Pediatr 2011;29: 294-9.

4. Gualano B, Sá Pinto AL, Perondi B, Leite Prado DM, Omori C, Almeida RT et al. Evidence for prescribing exercise as treatment in pediatric rheumatic diseases. Autoimmun Rev 2010;9:569-73.

5. Eccleston C, Palermo TM, Williams AC, Lewandowski A, Morley S. Psychological o conhecimento do local correto do ponto de acupuntura, a massagem prévia do local correto antes da inserção e o uso mínimo de agulhas em pontos de ampla ação e em locais visíveis para a criança facilitam o início do tratamento. A acupuntura não costuma ser um procedimento doloroso. Pacientes relatam apenas uma sensação de picada ou pressão no local de inserção da agulha.

Em muitos centros de referência em dor pediátrica, a acupuntura é uma realidade. Alguns exemplos são as clínicas de dor do Children's Hospital de Boston e do Children's Hospital de Los Angeles, nas quais os especialistas participam da equipe multiprofissional de tratamento e são realizadas reuniões regulares para a discussão dos casos clínicos.

O desafio para a abordagem de crianças e adolescentes com dor é grande, tanto para os casos agudos quanto crônicos. A acupuntura e a medicina ocidental podem e devem ser consideradas complementares. Certamente, muitos pacientes se beneficiam de ambas, sendo o controle da dor o objetivo final. Faz-se necessária uma abordagem holística do indivíduo, uma vez que a dor tem origem multifatorial (e não apenas física/nociceptiva) e os aspectos emocionais e sociais devem ser considerados e enfrentados. therapies for the management of chronic and recurrent pain in children and adolescents. Cochrane Database Syst Rev 2009;(2):CD003968.

6. Kundu A, Berman B. Acupuncture for pediatric pain and symptom management. Pediatr Clin North Am 2007;54:885-9.

7. Palermo TM, Eccleston C, Lewandowski AS, Williams AC, Morley S. Randomized controlled trials of psychological therapies for management of chronic pain in children and adolescents: an updated meta-analytic review. Pain 2010;148:387-97.

8. Sherry DD. An overview of amplified musculoskeletal pain syndromes. J Rheumatol 2000;58 (Suppl):44-8.

9. Dias MH, Amaral E, Pai HJ, Tsai DT, Lotito AP, Leone C et al. Acupuntura em adolescentes com fibromialgia juvenil. Rev Paul Pediatr 2012;30:6-12 